\title{
BMJ Open Documenting and explaining the HIV decline in east Zimbabwe: the Manicaland General Population Cohort
}

\author{
Simon Gregson, ${ }^{1,2}$ Owen Mugurungi, ${ }^{3}$ Jeffrey Eaton, ${ }^{1}$ Albert Takaruza, ${ }^{2}$ \\ Rebecca Rhead, ${ }^{1}$ Rufurwokuda Maswera, ${ }^{2}$ Junior Mutsvangwa, ${ }^{2}$ Justin Mayini, ${ }^{2}$ \\ Morten Skovdal, ${ }^{4}$ Robin Schaefer, ${ }^{1}$ Timothy Hallett, ${ }^{1}$ Lorraine Sherr, ${ }^{5}$ \\ Shungu Munyati, ${ }^{2}$ Peter Mason, ${ }^{2}$ Catherine Campbell, ${ }^{6}$ Geoffrey P Garnett, ${ }^{1}$ \\ Constance Anesu Nyamukapa ${ }^{1,2}$
}

To cite: Gregson S,

Mugurungi 0, Eaton J, et al. Documenting and explaining the HIV decline in east Zimbabwe: the Manicaland General Population Cohort. BMJ Open 2017;7:e015898. doi:10.1136/ bmjopen-2017-015898

- Prepublication history and additional material are available online. To view these files please visit the journal online (http://dx. doi.org/10.1136/bmjopen-2017015898).

Received 8 January 2017 Revised 10 April 2017 Accepted 27 April 2017

\section{CrossMark}

${ }^{1}$ Department of Infectious Disease Epidemiology, Imperial College London, London, UK ${ }^{2}$ Biomedical Research and Training Institute, Harare, Zimbabwe

${ }^{3}$ Zimbabwe Ministry of Health and Child Care, Harare,

Zimbabwe

${ }^{4}$ University of Copenhagen, Copenhagen, Denmark

${ }^{5}$ University College London, London, UK

${ }^{6}$ London School of Economic and Political Science, London, UK

Correspondence to Professor Simon Gregson; sajgregson@aol.com

\section{ABSTRACT}

Purpose The Manicaland cohort was established to provide robust scientific data on HIV prevalence and incidence, patterns of sexual risk behaviour and the demographic impact of HIV in a sub-Saharan African population subject to a generalised HIV epidemic. The aims were later broadened to include provision of data on the coverage and effectiveness of national HIV control programmes including antiretroviral therapy (ART). Participants General population open cohort located in 12 sites in Manicaland, east Zimbabwe, representing 4 major socioeconomic strata (small towns, agricultural estates, roadside settlements and subsistence farming areas). 9,109 of 11,453 (79.5\%) eligible adults (men 1754 years; women $15-44$ years) were recruited in a phased household census between July 1998 and January 2000. Five rounds of follow-up of the prospective household census and the open cohort were conducted at 2-year or 3-year intervals between July 2001 and November 2013. Follow-up rates among surviving residents ranged between $77.0 \%$ (over 3 years) and $96.4 \%$ ( 2 years).

Findings to date HIV prevalence was $25.1 \%$ at baseline and had a substantial demographic impact with 10 -fold higher mortality in HIV-infected adults than in uninfected adults and a reduction in the growth rate in the worst affected areas (towns) from $2.9 \%$ to $1.0 \%$ pa. HIV infection rates have been highest in young adults with earlier commencement of sexual activity and in those with older sexual partners and larger numbers of lifetime partners. HIV prevalence has since fallen to $15.8 \%$ and HIV incidence has also declined from 2.1\% (1998-2003) to $0.63 \%(2009-2013)$ largely due to reduced sexual risk behaviour. HIV-associated mortality fell substantially after 2009 with increased availability of ART.

Future plans We plan to extend the cohort to measure the effects on the epidemic of current and future HIV prevention and treatment programmes. Proposals for access to these data and for collaboration are welcome.

\section{INTRODUCTION}

The Manicaland general population open cohort HIV sero-survey (Manicaland cohort) was set up in 1998 by researchers from
Strengths and limitations of this study

- The Manicaland cohort is one of a handful of longrunning, large-scale general population HIV serosurveys conducted in countries in sub-Saharan Africa with widespread epidemics that constitute a key resource for evaluating the populationlevel impact of HIV prevention and treatment programmes.

- The current data span the period 1998 to 2013 during which Zimbabwe experienced one of the largest HIV epidemics in the world and was almost unique in sub-Saharan Africa in achieving a substantial national decline in HIV prevalence largely caused by reductions in sexual risk behaviour. The study data also cover periods prior to, during and following the roll-out of prevention of mother-tochild transmission services (introduced in Zimbabwe from 2002) and antiretroviral treatment services (from 2004 with rapid scale-up from 2009).

- The study data include comprehensive and consistent measurements of trends in HIV prevalence, HIV incidence, HIV-associated and all-cause mortality, sexual risk behaviours, healthseeking behaviours, and in the coverage and effects of national HIV control programmes over time. The study also includes parallel measurement of trends in HIV prevalence among pregnant women attending local antenatal clinics which permits assessment of biases in the primary source of routine HIV surveillance data used by countries and Joint United Nations Programme on HIV/AIDS to produce national and regional HIV estimates.

- Findings from the study are generalisable to Zimbabwe as a whole and data are available on their wider generalisability.

- Limitations of the cohort include the age limit (55 years) for participation, changes in eligibility criteria across rounds, and long intervals (2-3 years) between rounds of follow-up such that short-term migrants may be missed and measurement of some key variables including mortality can be subject to recall and misclassification bias. 
Imperial College London and the Biomedical Research and Training Institute (BRTI) with funding from the Wellcome Trust. Findings from an earlier study (1993-1996) had shown that HIV was spreading extensively in rural areas of eastern Zimbabwe, and was associated with large increases in mortality. ${ }^{1}$ The new cohort was established to provide robust scientific data on HIV prevalence and incidence within a general population sample, on local patterns of sexual behaviour and their role in the spread of HIV, and on the mortality and wider demographic impact of HIV in a range of different settings in Manicaland, Zimbabwe's eastern province.

Following an initial pilot study, ${ }^{2}$ in the first two rounds of the cohort study, a two-arm cluster-randomised controlled trial was conducted of a peer education, condom distribution and syndromic management of sexually transmitted infections (STIs) intervention in female sex workers and male clients to reduce the spread of HIV infection. The trial found that this intervention was not effective in reducing HIV incidence within the general population. ${ }^{3}$

In subsequent rounds of the cohort survey, the research aims were extended to include investigation of the temporal dynamics of the HIV epidemic, the social determinants of HIV, and the coverage and effectiveness of national HIV control programmes, including antiretroviral therapy (ART) services introduced in the mid-2000s.

\section{COHORT DESCRIPTION}

\section{Study design and location}

The study is designed as a stratified General Population Open Cohort HIV Sero-Survey and is located in three districts (Mutasa, Makoni and Nyanga) of Manicaland province, which runs along Zimbabwe's eastern border with Mozambique (figure 1A).

To accommodate the two-arm cluster-randomised trial, a stratified design was chosen with six pairs of sites (figure 1B) matched on socioeconomic criteria. Consequently, the Manicaland cohort was drawn from two small towns (Nyanga and Nyazura), four agricultural estates (Katiyo and Eastern Highlands tea estates and Selborne and Sheba forestry plantations), two roadside settlements (Watsomba and Nyabadza/Nyahukwe), and four subsistence farming areas (Bonda, Honde, St. Theresa's and St. Killian's missions).

The central coordinates of the component study locations (rural village markets, estate compounds and urban locations) have been mapped using handheld global positioning system devices (figure 1C). Overall, the study sites are located between latitudes $-18.07^{\circ}$ and $-18.85^{\circ}$ and longitudes $31.93^{\circ}$ and $33.04^{\circ}$, an average distance of $180.8 \mathrm{~km}$ (range: $126.1-219.6 \mathrm{~km})$ and $58.9 \mathrm{~km}(13.3-$ $99.3 \mathrm{~km}$ ) from Harare and Mutare, the national and provincial capitals, and encompass a combined area of $8184 \mathrm{~km}^{2}$.

The study areas are located in the Eastern Highlands region of Zimbabwe (average altitude approximately $1300 \mathrm{~m}$ ) and are predominantly rural but benefit from a temperate climate (quite hot with rains between October and March; cool and dry from May to August) with generally relatively good rainfall (average temperature and annual rainfall $\mathrm{c} 25^{\circ}$ and $\mathrm{c} 1000 \mathrm{~mm}$ ) and fertile soils. The principal crops include maize, sorghum, finger millet, yams, cotton, tea, bananas, avocados, sugarcane and other fruits. Most local people also grow vegetables and keep cattle, goats and chicken.

\section{Eligibility criteria and participation and follow-up rates}

The baseline census and survey were conducted in a phased manner (one site at a time) between July 1998 and January 2000. In the census, a household was defined as a group of people who regularly eat together from the same cooking pot. Regular household members aged 17-54 years for men and 15-44 years for women-the ages of expected highest HIV incidence-were eligible for enrolment into the cohort (figure 2). However, participation in the cohort was restricted to a maximum of one member of each marital group (ie, a man and his wife or wives), selected at random, in order to maximise statistical power for the trial of the peer education and STI treatment intervention. Local village community workers were employed as guides to assist in locating participants. Where eligible individuals were unavailable for interview at the first household visit, appointments and up to two additional visits were made.

The second round of the open cohort survey was conducted between August 2001 and July 2003. All baseline respondents and individuals who had aged into the qualifying age range were eligible for this round. In-migrants in the 3-year intersurvey period and visitors were eligible for enrolment in the last eight sites. The third round ran from August 2003 to August 2005. Eligibility for the cohort was extended to include all men and women aged 15-54 years and the restriction to one member of each marital group was lifted. The fourth round ran from August 2006 to November 2008. All households were eligible for enumeration in the census and the same age criteria were used but follow-up and recruitment into the cohort were limited to members of a random sample of two-thirds of households due to funding constraints. The same eligibility criteria were applied in round 5, which ran from October 2009 to July 2011, and in round 6, which ran from July 2012 to November 2013. In round 6 , four sites were dropped from the study, again due to funding constraints.

In the household censuses, the overall response rate was $98.2 \%(8233 / 8386)$ at baseline $(1998-2000)$, $97.1 \%(6982 / 7189)$ in round $2(2001-2003), 95.4 \%$ $(9322 / 9773)$ in round $3,93.7 \%(11865 / 12668)$ in round $4,98.0 \%(13180 / 13453)$ in round 5 and $90.9 \%$ $(8116 / 8931)$ in round 6 (2012-2013) (see online supplementary table $\mathrm{S} 1$ ). This estimate for the response rate in the baseline census is an overestimate because fears that the researchers were Satanists-because they were asking for blood specimens thought to be used by Satanistscaused difficulties in identifying households. From the 


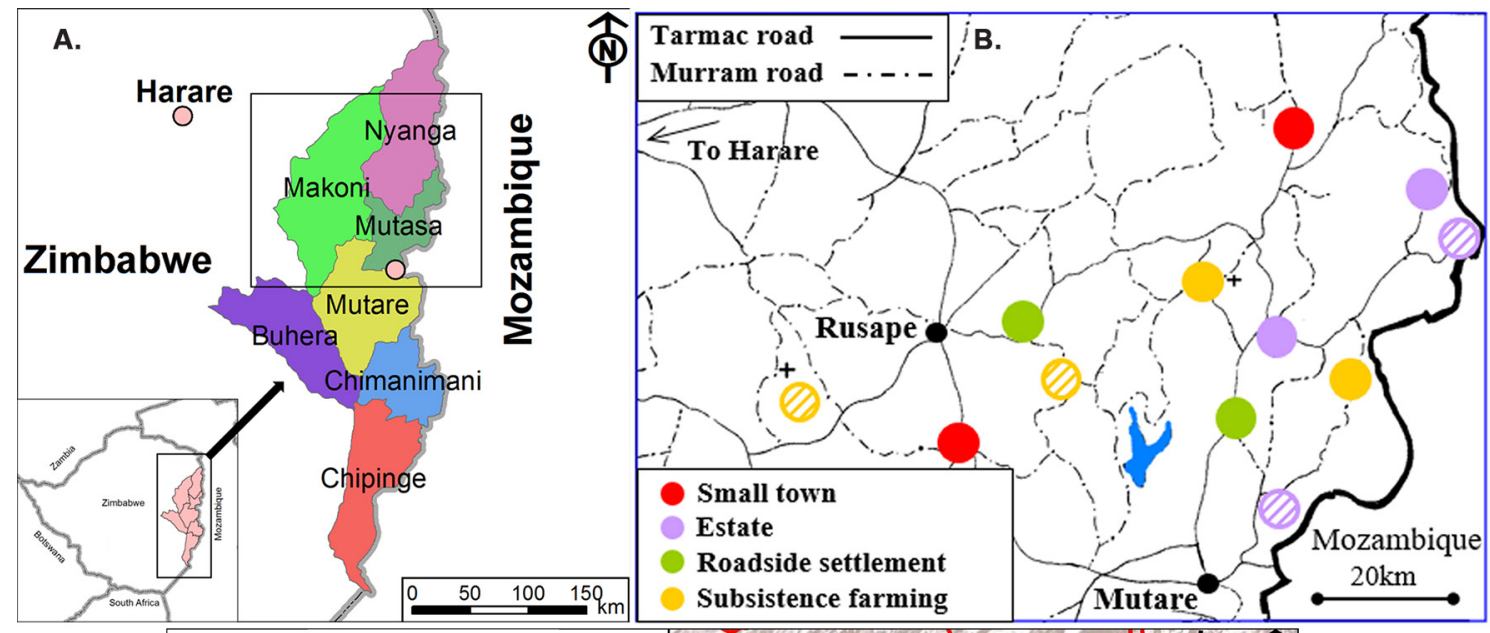

c. Legend

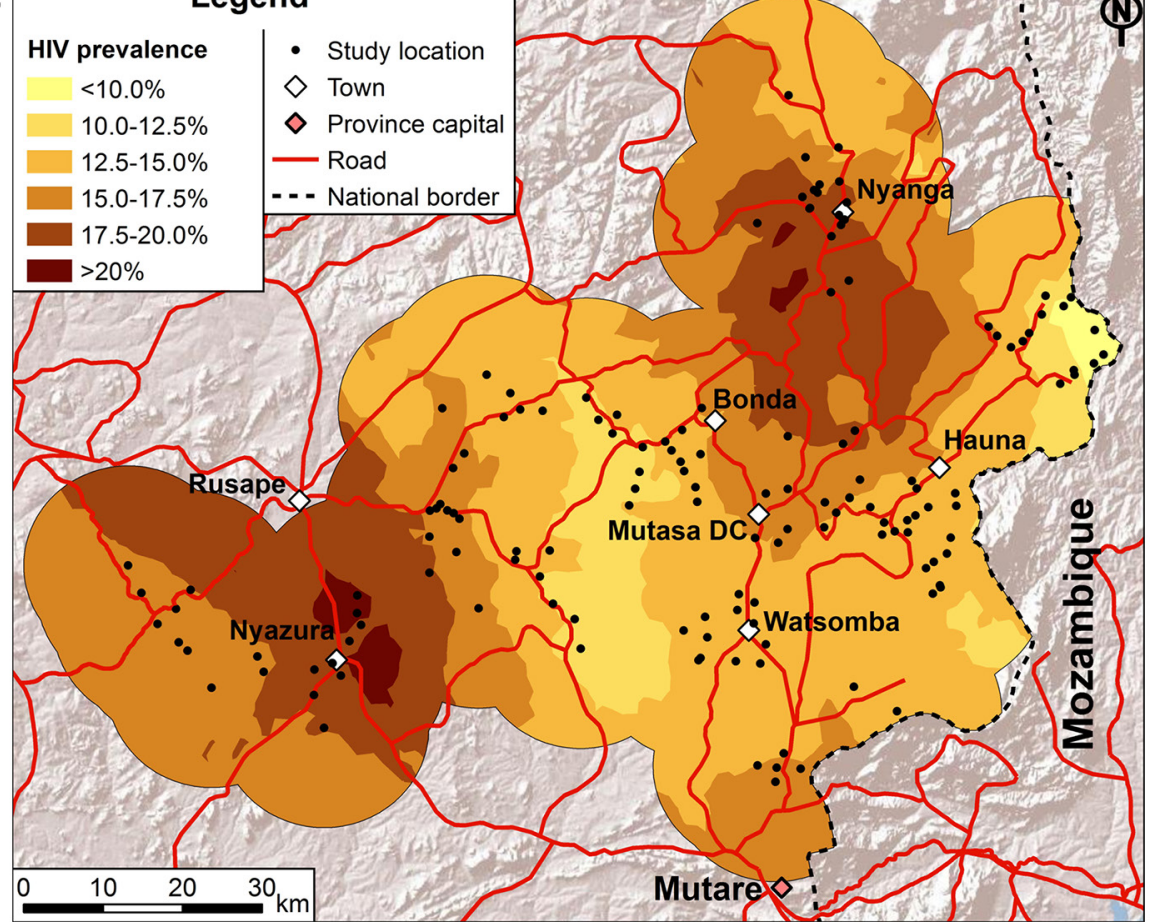

Figure 1 (A) Location of the study districts in Manicaland province, east Zimbabwe; (B) map showing the 12 study areas in Manicaland province with the four sites excluded from round 6 shown with shading; (C) map of HIV prevalence across the study areas showing the study villages, estate compounds and urban locations at round 5 (2009-2011).

third round onwards, a steady increase in numbers of households has been observed reflecting reductions in these Satanist fears and growth in the population. The escalating economic crisis and a government initiative in 2005 to clean up the urban areas contributed to the increase in household numbers in round 4 (2006-2008). No difference in response rates was seen between households selected and not selected for individual interviews in rounds 4 to 6 (see online supplementary table S1).

At baseline, 11453 individuals were eligible for the study, of whom $79.5 \%$ (9109) participated (figure 2, see online supplementary figure S2). In subsequent rounds, using the same age ranges for each sex for comparison (17-54 years for men; 15-44 years for women), overall participation rates have been similar except in round
6 when the overall rate fell to $73.0 \%$. The cohort size has varied between 6269 in round 2 (2001-2003) and 13196 in round 3 (2003-2005) reflecting, primarily, the changes in eligibility criteria between rounds. Participation rates generally have been higher in women than in men (see online supplementary table S2). Direct refusal rates are consistently low $(<5 \%)$, most non-participation being due to temporary absences from the household reflecting the high population mobility found in Zimbabwe.

The follow-up rate among all members of the cohort in the preceding round has varied between rounds from $47.0 \%$ (2006-2008 to 2009-2011) and 60.6\% (2001-2003 to 2003-2005) (figure 2, see online supplementary table S1). However, most loss to follow-up comprises previous members of the cohort who ceased to be eligible due to 


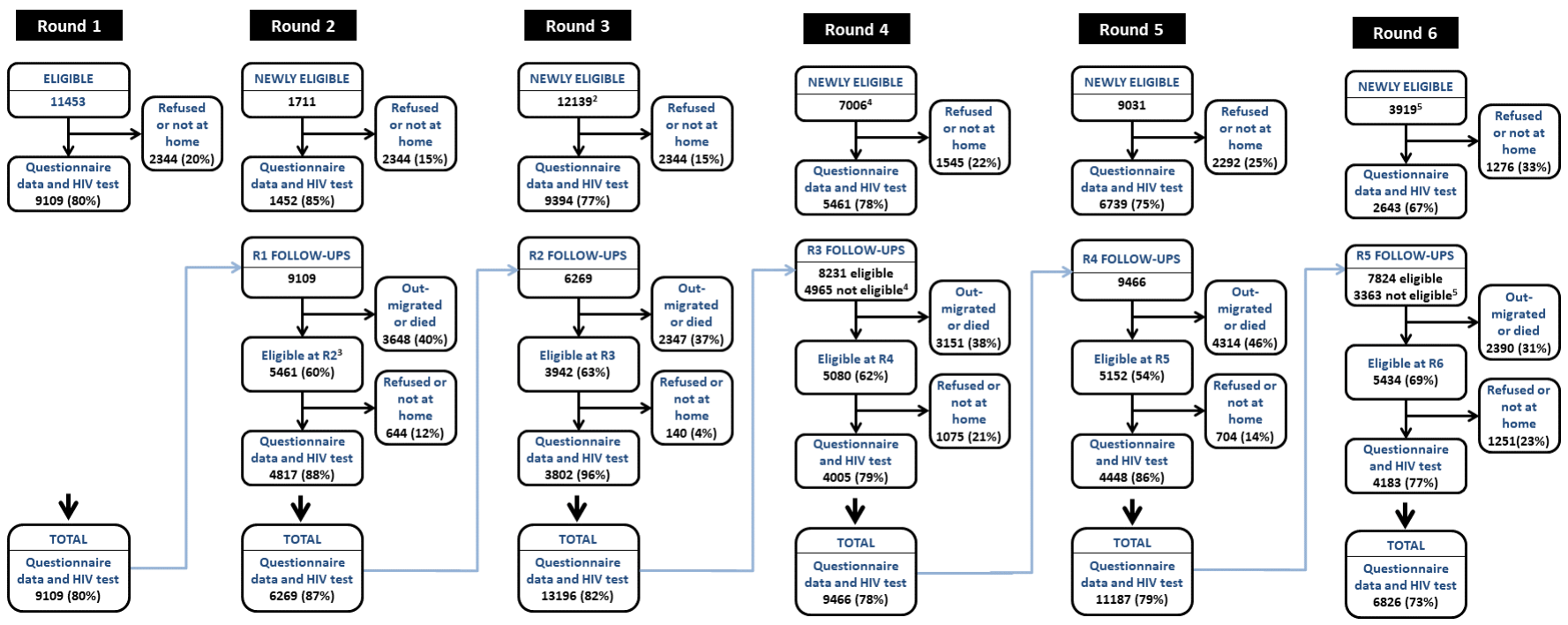

Figure 2 Flow diagram showing individual participation rates and follow-up rates in the cohort by survey round. Notes: (1) Participation and follow-up rates based on eligibility criteria at round 1: men aged 17-54, women aged 15-44; regular members of households in the study areas; stayed in the household at least 4 nights in the last month. (2) In rounds 1 and 2, a maximum of one member per marital grouping was selected at random for interview; this restriction was dropped from round 3 onwards. (3) In round 2, individuals who migrated into a household since round 1 were only treated as eligible from site 5 (out of 12 sites). (4) From round 4, eligibility for individual interviews was restricted to individuals from a random sample of two-thirds of enumerated households. (5) In round 6, the number of study sites was reduced from 12 to 8 (2 agricultural estates and 2 subsistence farming areas were dropped). R1, R2, ... indicate round numbers in the cohort survey.

death or out-migration from the study areas. Among those who remained eligible, cohort follow-up rates have been high, ranging from $77.0 \%$ (2009-2011 to 2012-2013) to 96.4\% (2001-2003 to 2003-2005). Follow-up rates have differed little between HIV-positive and HIV-negative individuals.

\section{Questionnaire data}

In the household census questionnaire, the location and identity of each household is recorded (table 1). Basic sociodemographic information is collected for each member of the household including information on the eligibility criteria for inclusion in the cohort for adults. In households enumerated in previous rounds of the census, details of individuals who stayed in the household at or following the last visit are recorded (even if they have since left the household) and details of their survival status and date of leaving the household (where applicable) are recorded. Information on moveable and immoveable household assets is also collected for use in measuring socioeconomic status using wealth indices. ${ }^{4}$

The individual respondent questionnaires used for the Manicaland cohort comprise sections on the respondent's own sociodemographic characteristics, the characteristics of up to four current spouses, the respondent's psychological health (since round 5-including all questions from the Shona Symptom Questionnaire, ${ }^{5}$ a locally validated common mental health inventory and WHO's Self-Report Questionnaire ${ }^{6}$ ), sexual relationships, health and access to treatment (since round 3), HIV knowledge and awareness (including exposure to HIV control programmes), and fertility and pregnancy histories (table 1). Dried blood spot (DBS) specimens have been collected for anonymised HIV testing for research purposes only as a requirement for participation at each round of the cohort. Free parallel voluntary HIV counselling and testing services were made available locally for cohort members during survey visits. ${ }^{7}$

For cohort members who passed away between rounds of the survey, verbal autopsy interviews were conducted with the deceased's primary caregiver. The questionnaire included questions on accidents, medical conditions and symptoms during the final illness, ${ }^{8}$ and on social and financial circumstances surrounding the death ${ }^{9}$ (table 1 ).

Unusual features of the Manicaland cohort include parallel HIV surveys among pregnant women attending antenatal (ANC) check-ups at local health clinics, conducted to obtain information in biases in routine HIV surveillance data ${ }^{10}$ (see online supplementary table S4); and six rounds of facility surveys conducted (2010-2016) to measure trends in local availability of HIV services. ${ }^{11}$

In the first five rounds of the Manicaland cohort, all interviews were conducted using paper questionnaires. In round 6 , the questionnaires for household census and individual cohort interviews were administered using HTC Smartphones using EpiCollect software. ${ }^{12}$ Copies of the study questionnaires are available from the Manicaland Centre for Public Health Research website (http://www.manicalandhivproject.org/questionnaires). The data from all rounds of the study are held in an SQL relational database developed for use in Microsoft Access. 
Table 1 Information collected in the household census, individual interviews, verbal autopsy interviews in the Manicaland Cohort Survey

\begin{tabular}{|c|c|c|c|}
\hline $\begin{array}{l}\text { Survey instrument } \\
\text { Variable(s) }\end{array}$ & $\begin{array}{l}\text { Survey } \\
\text { rounds* }\end{array}$ & Scope of question & Specific information \\
\hline \multicolumn{4}{|l|}{ Household census } \\
\hline Household ID & All & Each household & District, village name, household head \\
\hline Household status & All & Each household & New or dissolved \\
\hline $\begin{array}{l}\text { Pre-existing household } \\
\text { members }\end{array}$ & All & Each household & $\begin{array}{l}\text { Name, relationship to household head, sex, age, } \\
\text { education, parents' survival status, member's survival } \\
\text { status, nights spent in household in the last month, } \\
\text { whether selected for interview }\end{array}$ \\
\hline New household members & All & Each household & As above plus date joined household \\
\hline Former household members & All & Each household & $\begin{array}{l}\text { Survival status, date and reason for leaving } \\
\text { household, current residence (for out-migrants) }\end{array}$ \\
\hline Household assets & All & Each household & $\begin{array}{l}\text { Water source, toilet type, house type, moveable } \\
\text { assets }\end{array}$ \\
\hline GPS coordinates & Round 6 & $\begin{array}{l}\text { Collected at village level } \\
\text { only }\end{array}$ & GPS coordinates for central market area \\
\hline
\end{tabular}

Individual interviews

Background characteristics All Random sample of adult Sex, age, parents' survival (<30years), education, household members $\dagger$ migration, religion, male circumcision, employment, substance use, marital history and status, participation in community groups

\begin{tabular}{|c|c|c|c|}
\hline Spouse characteristics & All & Up to four spouses & $\begin{array}{l}\text { Age, age at marriage, cohabitation, education, } \\
\text { employment, HIV test and disclosure, migration, } \\
\text { religion, male circumcision }\end{array}$ \\
\hline Psychological health & $\begin{array}{l}\text { Rounds } 5 \\
\text { to } 6\end{array}$ & All selected adults & $\begin{array}{l}\text { Variables for Shona Symptom Questionnaire and } \\
\text { WHO Questionnaire }\end{array}$ \\
\hline Sexual relationships & All & All selected adults & $\begin{array}{l}\text { Age at first sex, regular/non-regular partners, condom } \\
\text { use, partner loops, concurrency, commercial/ } \\
\text { transactional sex, informal confidential voting } \\
\text { interviews used for literate participants }\end{array}$ \\
\hline $\begin{array}{l}\text { Health and access to } \\
\text { treatment }\end{array}$ & $\begin{array}{l}\text { Rounds } 3 \\
\text { to } 6\end{array}$ & All selected adults & $\begin{array}{l}\text { General health, healthcare behaviour, STDs, HIV } \\
\text { testing, disclosure, CD4 counts, ART initiation / } \\
\text { adherence, side effects, palliative care }\end{array}$ \\
\hline HIV awareness and impact & All & All selected adults & $\begin{array}{l}\text { Knowledge, risk perception, self efficacy, stigma, } \\
\text { masculinity, exposure to HIV prevention }\end{array}$ \\
\hline Fertility history & All & All women & $\begin{array}{l}\text { Sex, date of birth, PMTCT uptake, survival status, } \\
\text { date of death }\end{array}$ \\
\hline Pregnancy history & All & $\begin{array}{l}\text { Current and recent } \\
\text { pregnancies (last 3years) }\end{array}$ & $\begin{array}{l}\text { Antenatal care, PMTCT uptake, infant diagnosis, } \\
\text { breastfeeding, postpartum amenorrhoea, sexual } \\
\text { abstinence, family planning }\end{array}$ \\
\hline HIV infection status & All & All selected adults & $\begin{array}{l}\text { Combaids HIV-1/HIV-2 dipstick test; potential } \\
\text { seroconversions, confirmed with Vironostika HIV } \\
\text { Uniform-II plus O }\end{array}$ \\
\hline \multicolumn{4}{|l|}{ Verbal autopsy interviews } \\
\hline Social circumstances & Round $2 \rightarrow$ & Deaths in the cohort & $\begin{array}{l}\text { Relationship of caregiver/respondent to deceased, } \\
\text { sex, age, date of death, HIV testing and ARV } \\
\text { treatment /adherence, history of deceased; } \\
\text { deceased's spouse's status, household impact }\end{array}$ \\
\hline Financial implications & & Deaths in the cohort & $\begin{array}{l}\text { Healthcare costs and funding contributions, impact } \\
\text { of illness, on employment, pension/termination } \\
\text { payments, widow's pension }\end{array}$ \\
\hline $\begin{array}{l}\text { Effects on deceased's } \\
\text { children }\end{array}$ & & $\begin{array}{l}\text { Births before and since } \\
\text { deceased's last interview }\end{array}$ & $\begin{array}{l}\text { Survival status, PMTCT, orphanhood, education, care } \\
\text { arrangements }\end{array}$ \\
\hline
\end{tabular}


Table 1 Continued

\begin{tabular}{llll}
\hline $\begin{array}{l}\text { Survey instrument } \\
\text { Variable(s) }\end{array}$ & $\begin{array}{l}\text { Survey } \\
\text { rounds* }\end{array}$ & Scope of question & Specific information \\
\hline $\begin{array}{l}\text { Medical conditions and } \\
\text { accidents }\end{array}$ & Deaths in the cohort & Accidents, homicide, suicide \\
$\begin{array}{l}\text { Maternal mortality } \\
\text { Final illness }\end{array}$ & Female deaths & Symptoms of maternal mortality \\
\hline
\end{tabular}

*Dates for rounds of the household census and individual cohort: round 1: July 1998 to January 2000; round 2: August 2001 to July 2003; round 3: August 2003 to August 2005; round 4: August 2006 to November 2008; round 5: October 2009 to July 2011; round 6: July 2012 to November 2013.

† Eligibility for the adult individual general population cohort: round 1: regular household members who had slept in the household at least four nights in the last month and had been resident in the household at the same time 1 year earlier, men aged 17-54 years and women aged 15-44 years limited to one member of a marital union selected at random (to maximise power in the embedded community randomised controlled trial of HIV prevention interventions); round 2: same criteria as in round 1 except that in-migrants were not eligible in the first four sites (Katiyo tea estate, Eastern Highlands tea estates, Bonda Mission, Honde Mission). In the remaining eight sites (and in all sites in subsequent rounds), individuals who stayed in households in the study areas the night before the census visit but who had not met the round 1 residence tests were treated as eligible for participation in the cohort; round 3: eligible age ranges extended to 15-54years for men and women; restriction to one member of each marital union dropped and residence criteria extended to all persons who slept in the household the previous night rounds 4 and 5: same criteria as in round 3 except that eligibility was limited to adults in a random sample of two-thirds of households in the household census; round 6: same criteria as in rounds 4 and 5 but restricted to eight sites: Eastern Highlands tea estate, Bonda Mission, Honde Mission, Selborne forestry estate, Nyazura, Nyanga, Watsomba, Nyabadza/Nyahukwe.

ART, antiretroviral therapy; PMTCT, prevention of mother-to-child transmission of HIV infection; STDs, sexually transmitted diseases.

\section{Data on HIV infection rates}

New participants in the cohort at each round provided DBS specimens that were tested for HIV infection at the BRTI laboratory in Harare, using a consistent testing strategy ${ }^{13}$ across all rounds of the survey. At each round of follow-up, HIV-negative individuals from the previous round were retested for HIV infection using newly collected DBS specimens and the same testing strategy. The HIV testing strategy used a dipstick dot-EIA test as the screening test (the ICL dipstick dot-EIA (ICL-HIV 1\&2 Dipstick, Thailand)) in round 1 and the Combaids dot-EIA (Combaids-HIV-1\&2 Dipstick, Span Diagnostics, India) in rounds 2 to 6 , and a third generation plate EIA (Abbott third generation HIV 1\&2 EIA (Abbott Laboratories, USA)) or Genelavia MIXT HIV1\&2 (Sanofi Diagnostics Pasteur SA, France) in rounds 1 and 2; Vironostika HIV Uniform II in rounds 3 to 6 ) as the confirmatory test. Where the test results from successive survey rounds indicated a seroconversion, the sample from the first of these rounds was retested to confirm the original negative result using the same dipstick dot-EIA test. Where this result remained negative, the plate EIA test was run on the DBS specimens from both rounds to confirm the results. BRTI laboratory test results were routinely evaluated in the Zimbabwe National Quality Assurance Programme.

The HIV incidence rates for each intersurvey period reported in this paper were estimated assuming that new infections between rounds occurred midway between the first and second interview dates.

\section{Characteristics of the study population}

The study population is comprised primarily of people who speak the Manyika dialect of Zimbabwe's majority Shona language. Most are also Christian, belonging to a large number of different Mission, Apostolic, Pentecostal and other spiritual churches. ${ }^{14}$ Customary marriage, based on payment of bride-wealth, is almost universal, and is often followed by a church wedding. Polygyny remains common in some Apostolic churches and people who practice traditional religion. ${ }^{15}$ As elsewhere in Zimbabwe, education levels are high compared with other countries in sub-Saharan Africa. ${ }^{1617}$

Just over half of the cohort is female reflecting the predominantly rural study areas (table 2 ). Over time, the cohort has aged somewhat (from a median of 25 years in round 1 to 27 years in round 5) and the proportion living on agricultural estates has fallen due to retrenchments on these estates reflecting increased mechanisation and the economic decline. The latter is also reflected in the large increase in unemployment between round $2(32.8 \%)$ and round $4(55.5 \%)$. However, education levels ${ }^{17}$ and the proportion of the cohort who are married have both increased due, in part, to the ageing of the cohort.

\section{FINDINGS TO DATE}

\section{HIV surveillance in a high HIV prevalence setting}

The pilot study provided important early evidence that, by the early 1990s, HIV prevalence had reached high levels $(23.3 \%)$ in the general population in rural areas of Zimbabwe. ${ }^{2}$ Up to this point, HIV prevalence had been found to be much higher in urban areas than in rural areas in most sub-Saharan African countries and the high prevalence in rural areas meant that Zimbabwe was faced with one of the world's largest HIV epidemics. The study also found extremely high prevalence in young women aged 15-24 years $(20.8 \%){ }^{2}$ In the Manicaland cohort, HIV prevalence was $25.1 \%$ at baseline (1998-2000) and fell steadily to $16.7 \%$ in round 5 and $15.8 \%$ in round 6 (table 2, figure 3 ). 
Table 2 Sociodemographic characteristics of cohort participants by survey round, Manicaland cohort, Zimbabwe, 1998-2013

\begin{tabular}{|c|c|c|c|c|c|c|}
\hline & 1998-2000 & 2001-2003* & 2003-2005 & 2006-2008 & 2009-2011 & 2012-2013† \\
\hline & $\mathbf{N}$ & $\mathbf{N}$ & $\mathbf{N}$ & $\mathbf{N}$ & $\mathbf{N}$ & $\mathbf{N}$ \\
\hline Number of participants & 9109 & 6269 & 13196 & 9466 & 11187 & 6826 \\
\hline \multicolumn{7}{|l|}{ Sex } \\
\hline Male & 4164 (45.7\%) & $2730(43.6 \%)$ & $5314(40.3 \%)$ & $3919(41.4 \%)$ & 4474 (40.0\%) & $2772(40.6 \%)$ \\
\hline Female & $4945(54.3 \%)$ & 3539 (56.4\%) & $7882(59.7 \%)$ & $5547(58.6 \%)$ & $6713(60.0 \%)$ & 4054 (59.4\%) \\
\hline \multicolumn{7}{|l|}{ Age (years) } \\
\hline 15-24 (17-24 for men) & $4300(47.2 \%)$ & 2765 (44.1\%) & $6039(45.8 \%)$ & 4075 (43.0\%) & 4394 (39.3\%) & 2444 (35.8\%) \\
\hline $25-34$ & $2630(28.9 \%)$ & $1820(29.0 \%)$ & $3973(30.1 \%)$ & $3072(32.5 \%)$ & 3633 (32.5\%) & $2263(33.2 \%)$ \\
\hline $35-44$ & $1832(20.1 \%)$ & $1408(22.5 \%)$ & $2639(20.0 \%)$ & $1912(20.2 \%)$ & $2622(23.4 \%)$ & $1741(25.5 \%)$ \\
\hline 45-54 (men only) & 347 (3.8\%) & $276(4.4 \%)$ & $545(4.1 \%)$ & $406(4.3 \%)$ & $538(4.8 \%)$ & $378(5.5 \%)$ \\
\hline \multicolumn{7}{|l|}{ Residence } \\
\hline Small towns & 1539 (16.9\%) & $978(15.6 \%)$ & $2174(16.5 \%)$ & $1578(16.7 \%)$ & $2010(18.0 \%)$ & $1759(25.8 \%)$ \\
\hline Agricultural estates & $3005(33.0 \%)$ & 2095 (33.4\%) & $4022(30.5 \%)$ & $2663(28.1 \%)$ & $2992(26.7 \%)$ & $1418(20.8 \%)$ \\
\hline Roadside settlements & $1530(16.8 \%)$ & $1090(17.4 \%)$ & $2493(18.9 \%)$ & 1789 (18.9\%) & $2246(20.1 \%)$ & $1917(28.1 \%)$ \\
\hline $\begin{array}{l}\text { Subsistence farming } \\
\text { villages }\end{array}$ & 3035 (33.3\%) & $2106(33.6 \%)$ & $4507(34.1 \%)$ & $3436(36.3 \%)$ & 3939 (35.2\%) & $1732(25.3 \%)$ \\
\hline \multicolumn{7}{|l|}{ Migrant status } \\
\hline In-migrant (<3years) & $2182(23.9 \%)$ & 788 (12.6\%) & $2282(17.4 \%)$ & $1716(18.1 \%)$ & $1299(11.6 \%)$ & 738 (10.8\%) \\
\hline Non-migrant & 6927 (76.1\%) & $5481(87.4 \%)$ & $10904(82.6 \%)$ & 7750 (81.9\%) & 9888 (88.4\%) & 6088 (89.2\%) \\
\hline \multicolumn{7}{|l|}{ School education } \\
\hline None & $271(3.0 \%)$ & $86(1.4 \%)$ & $9(0.1 \%)$ & $0(0.0 \%)$ & $2(0.0 \%)$ & $48(0.7 \%)$ \\
\hline Primary & $3276(36.0 \%)$ & $1970(31.4 \%)$ & $3692(28 \%)$ & $2150(22.7 \%)$ & $2272(20.3 \%)$ & $1314(19.2 \%)$ \\
\hline Secondary & $5394(59.2 \%)$ & 4097 (65.4\%) & $8954(67.8 \%)$ & $6968(73.6 \%)$ & 8560 (76.5\%) & 5265 (77.1\%) \\
\hline Higher & $164(1.8 \%)$ & $53(0.8 \%)$ & $198(1.5 \%)$ & $180(1.9 \%)$ & $245(2.2 \%)$ & $154(2.3 \%)$ \\
\hline Missing & 4 (0.0\%) & $63(1.0 \%)$ & $343(2.6 \%)$ & $168(1.8 \%)$ & $108(1.0 \%)$ & 45 (0.7\%) \\
\hline \multicolumn{7}{|l|}{ Marital status } \\
\hline Single & 3391 (37.2\%) & 2274 (36.3\%) & $4431(33.6 \%)$ & $3128(33.0 \%)$ & $3149(28.2 \%)$ & $1782(26.1 \%)$ \\
\hline Married & 4537 (49.8\%) & 3280 (52.3\%) & $7110(53.9 \%)$ & $5138(54.3 \%)$ & $6776(60.6 \%)$ & 4277 (62.6\%) \\
\hline Divorced or separated & 762 (8.4\%) & 393 (6.3\%) & $996(7.5 \%)$ & $621(6.6 \%)$ & $640(5.7 \%)$ & $470(6.9 \%)$ \\
\hline Widowed & 405 (4.5\%) & 309 (4.9\%) & $636(4.8 \%)$ & $456(4.8 \%)$ & $546(4.9 \%)$ & $279(4.1 \%)$ \\
\hline Missing & $14(0.1 \%)$ & $13(0.2 \%)$ & $23(0.2 \%)$ & $123(1.3 \%)$ & $76(0.7 \%)$ & $18(0.3 \%)$ \\
\hline \multicolumn{7}{|l|}{ Employment status } \\
\hline Formal sector & 2344 (25.7\%) & 1725 (27.5\%) & $3037(23.0 \%)$ & 1982 (20.9\%) & $1942(17.4 \%)$ & $1246(18.2 \%)$ \\
\hline Informal sector & 2920 (32.1\%) & 1493 (23.8\%) & 2568 (19.4\%) & $1015(10.7 \%)$ & 1620 (14.5\%) & $795(11.7 \%)$ \\
\hline Unemployed & 3076 (33.8\%) & 2059 (32.8\%) & $5870(44.5 \%)$ & 5252 (55.5\%) & 6225 (55.6\%) & 3786 (55.5\%) \\
\hline Student & 755 (8.3\%) & 992 (15.8\%) & $1698(12.9 \%)$ & 1194 (12.6\%) & 1394 (12.5\%) & 824 (12.1\%) \\
\hline Missing & $14(0.1 \%)$ & $0(0.0 \%)$ & $23(0.2 \%)$ & $23(0.2 \%)$ & $6(0.0 \%)$ & 175 (2.6\%) \\
\hline \multicolumn{7}{|l|}{ HIV-positive } \\
\hline 12 original sites & 2127 (23.4\%) & 1337 (21.3\%) & $2533(19.2 \%)$ & 1657 (17.5\%) & 1795 (16.1\%) & - \\
\hline 8 sites in round 6 & 1502 (25.1\%) & 964 (23.3\%) & 1784 (19.9\%) & $1230(18.0 \%)$ & $1306(16.7 \%)$ & 1065 (15.8\%)‡ \\
\hline
\end{tabular}

To provide a consistent comparison across rounds of the cohort survey, these data are shown throughout for men aged 17-54years and women aged 15-44 years who were regular household members and stayed in the household for at least 4 nights in the last month before the interview.

*In round 2, individuals who had migrated into a household in the study areas since baseline were only treated as eligible from site 5 (out of 12 sites).

†In round 6, the number of study sites was reduced from 12 to 8 (2 agricultural estates and 2 subsistence farming areas were dropped). ‡Sixty missing cases due to indeterminate HIV test results. 


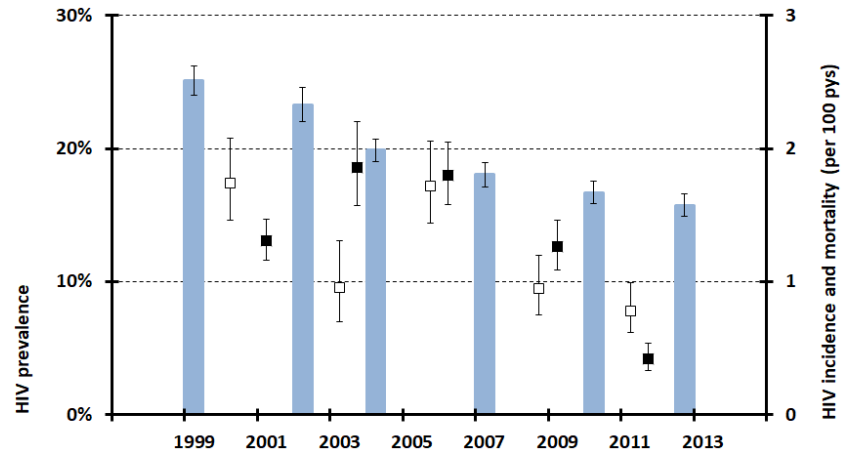

Figure 3 Trends in HIV prevalence (histogram), HIV incidence (open squares) and all-cause mortality (solid squares) in men aged 17-54 years and women aged 15-44 years resident in the eight sites included in all six rounds of the Manicaland general population open cohort sero-survey, Manicaland, Zimbabwe, 1998-2013. Whiskers indicate 95\% $\mathrm{Cl}$. Pyrs indicates person years.

In the late 1980s, Zimbabwe established a national HIV surveillance system based on unlinked anonymous testing of pregnant women attending ANC check-ups. ${ }^{18} 19$ The Manicaland Study, using data from its parallel general population cohort and ANC HIV prevalence surveys, has contributed information on the extent and causes of bias in ANC surveillance data on levels and trends in HIV prevalence ${ }^{1020-22}$ which have been used to develop the methods used in Zimbabwe and internationally to produce national HIV estimates. ${ }^{23-26}$

\section{Sexual behaviour, migration and the spread of HIV infection}

Understanding the role of sexual behaviour in the spread of HIV infection has been hampered by reporting biases in the data. ${ }^{27}$ In the Manicaland cohort, we developed an informal confidential voting interview method to reduce social desirability bias which has produced epidemiologically plausible results. ${ }^{28}$ Data from the cohort were used to provide a detailed description of patterns of sexual risk behaviour in eastern Zimbabwe and their associations with HIV infection ${ }^{1329}$ and of changes in behaviour over time (table 3). In particular, the data showed that large age differences between sexual partners were common in the study population (median difference 6 years for women aged 15-24 years; IQR 4-9 years) and were associated with increased risk of HIV infection in young people. ${ }^{29}$ Using a mathematical model, we found that age differences between men and women in sexual partnerships are unlikely to affect the scale of HIV epidemics but can explain the large female-male ratios of HIV infection found in young adults in sub-Saharan African populations. ${ }^{31}$ Data from the cohort provided evidence that medical injections are not a major contributor to new HIV infections in generalised epidemics. ${ }^{32}$

The Manicaland cohort provided the first evidence for declines in HIV prevalence occurring within the general population in Zimbabwe associated with reductions in sexual risk behaviours. ${ }^{13}$ In other studies, data from the cohort have been used to assess the effectiveness of national HIV prevention programmes in reducing sexual risk behaviour. ${ }^{33-35}$

The inter-relationships between migration and HIV in Manicaland are complex and not fully understood. ${ }^{36} 37$ However, while high internal population mobility may have driven the early spread of HIV infection into and within rural areas of Zimbabwe ${ }^{2-}$ and therefore contributed to the size of the national epidemic - the subsequent extensive out-migration from Zimbabwe was probably not a major factor in the decline in HIV prevalence that occurred from the late 1990s. ${ }^{1638}$

\section{The demographic impact of a generalised HIV epidemic}

Data from the Manicaland cohort were used to test early mathematical model predictions that HIV epidemics could eliminate the high rates of natural population increase ( $\geq 3 \%$ per annum) seen in sub-Saharan African countries in the 1980s. ${ }^{39-41}$ By the late 1990s, adult mortality in Manicaland was much higher among HIV-infected individuals (82/1000 person-years) than in uninfected individuals (7.2/1000 person-years) and the demographic impact was dramatic. However, even in the worst affected areas (towns with HIV prevalence of $33 \%$ ), population growth remained positive, falling by two-thirds from $2.9 \%$ to $1.0 \%{ }^{42}$ Using the cohort data, we demonstrated substantial reductions in fertility among HIV-infected women $^{43}$ and large increases in orphanhood ${ }^{44}$ (and associated risks of HIV infection and poor health in orphaned adolescents) ${ }^{45-47}$. Increases in coverage of ART from $2.3 \%$ in $2006-2008$ to $23.4 \%$ in $2009-2011$ and $38.2 \%$ in $2012-2013$ reduced death rates (figure 3$)^{48}$ but, as yet, have not prevented HIV-associated subfertility within the general population. ${ }^{22}$

\section{The role of social capital in HIV control in Zimbabwe}

Several studies have used data from the Manicaland cohort and, in some cases, qualitative data from the same populations to improve understanding of the underlying socioeconomic drivers of the spread of HIV infection. These have included studies on poverty and the influence of economic crises on patterns of HIV risk, ${ }^{49}$ and on patterns and effects of HIV stigma, ${ }^{50}$ masculinity, ${ }^{51} 52$ religion, ${ }^{1453}$ and female sex work. ${ }^{54}$ An unusual feature has been the innovative mixed-methods research done to describe local patterns of social capital. Strong evidence was found for associations between female participation in a range of different types of local community groups and reductions in HIV risk $^{55}$ and faster uptake of new services including HIV testing and prevention of mother-to-child transmission of HIV services. ${ }^{56}$ The research developed the notion of HIV-competent communities ${ }^{57}$ and highlighted the importance of community leadership and participation as a key factor in the success of HIV control interventions. ${ }^{589}$

A full list of publications is available from the Manicaland Centre for Public Health Research website (see above). 


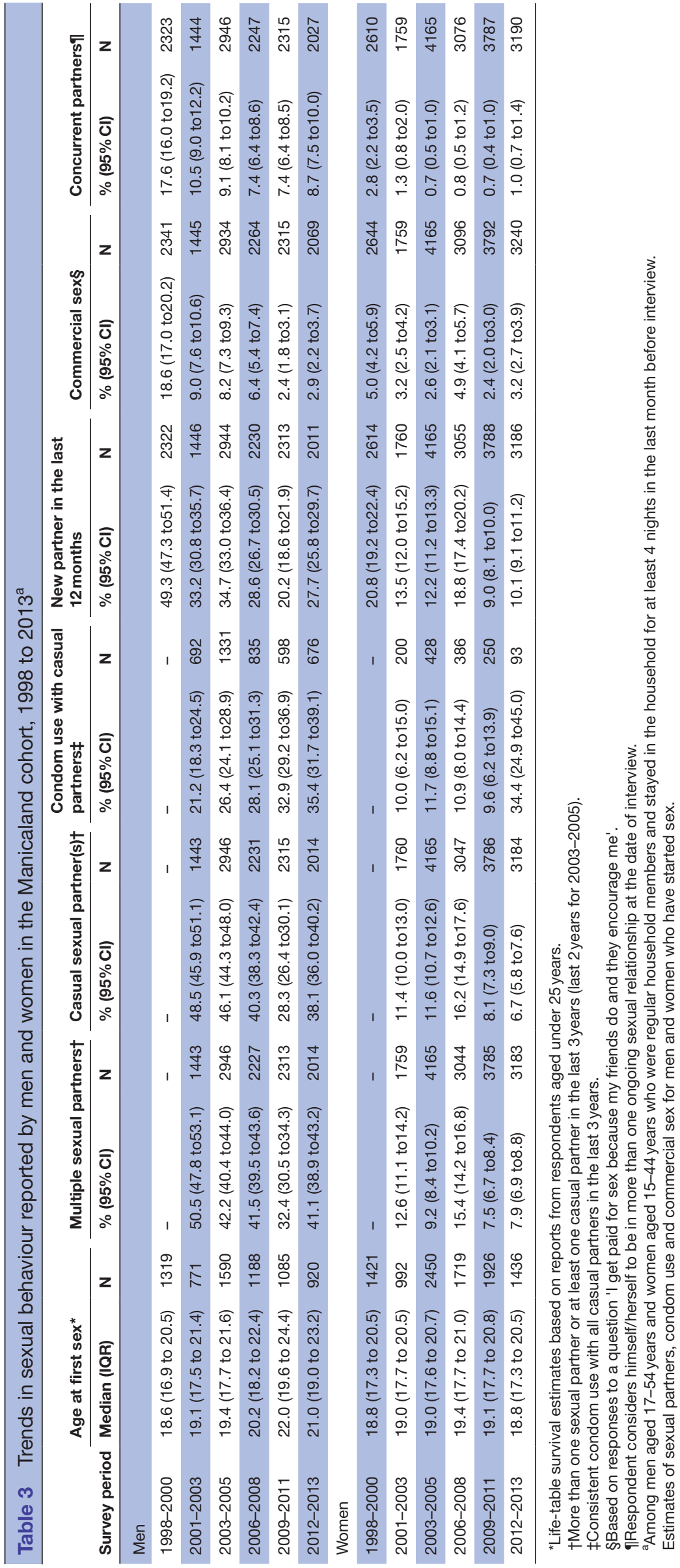




\section{STRENGTHS AND LIMITATIONS}

The Manicaland cohort is one of the handful of long-running, large-scale, General Population HIV Sero-Surveys conducted in sub-Saharan African countries with widespread epidemics that constitute a major resource for evaluating the population-level impact of HIV control strategies. ${ }^{60}$ A major strength of the Manicaland cohort is its comprehensive and consistent approach to measurement of trends in HIV prevalence, HIV incidence, HIV-associated mortality and all-cause mortality, sexual risk behaviours and health-seeking behaviours, and the coverage and effects of national HIV control programmes over time. The study is also unusual in its inclusion of a parallel ANC Survey, ${ }^{22}$ in its use of validated methods to improve the quality of data on sexual behaviour ${ }^{61}$ and in its use of mathematical models in interpreting the wider implications of the findings. ${ }^{3162}$

A weakness of studies that focus on localised areas can be difficulty in establishing whether findings are generalisable to the national population. In the Manicaland cohort, this has been addressed partially by including four of the main socioeconomic strata found in Zimbabwe. Triangulation of results with data from national sources shows that the overall levels and trends in the HIV epidemic observed in the study sites have been similar to those seen nationally. ${ }^{16} 6364$ The broader generalisability of the data on sexual behaviour patterns and trends has been explored in collaborative work with other general population studies in sub-Saharan Africa in the ALPHA network ${ }^{65}{ }^{66}$ and through studies using mathematical models. ${ }^{67}$ Specific weaknesses of the cohort that we hope to address in the future include the age limit (55 years) for participation, and the length of (18-24 months) and time intervals between (2-3years) rounds of follow-up. The latter means that individuals who move into and out of the study areas between rounds of the survey, may be missed and that measurement of some key variables including mortality can be subject to recall and misclassification bias.

\section{COLLABORATIONS AND FUTURE DIRECTIONS}

The Manicaland cohort has provided a valuable resource and platform for the design and implementation of a number of trials of HIV control interventions and collaborative projects led by independent researchers. These include trials of peer education among female sex workers and their clients (1998-2003) ${ }^{3}$ and conditional and unconditional cash transfers to support orphans and vulnerable children (2009-2011) ${ }^{68}$ studies on HIV and migration, ${ }^{37} 69$ and innovative studies on HIV competent schools $^{7071}$ and on patterns of social contacts that influence the spread of infectious diseases in children. ${ }^{72}$

Subject to funding availability, we plan to extend the cohort to provide data on the implementation and impact of current and future HIV control programmes including primary prevention interventions and programmes to address major comorbidities associated with the ageing of
HIV epidemics. We would welcome proposals for further collaborative projects related to this work.

Acknowledgements The authors thank the Zimbabwe Ministry of Health and Child Care and Zimbabwe National AIDS Council at national, provincial and district levels for their kind support and collaboration for the study. The authors also thank the following current and former members of the study leadership and management team: Saina Adamson (deceased), Roy M Anderson FRS, Lilian Banda, Stephen K Chandiwana (deceased), Godwin Chawira, Louis Chisvo, Ide Cremin, Elijah Dauka, Sabada Dube, Jocelyn Elmes, Noah Kadzura, Memory Kakowa, James Lewis, Ben Lopman, Edith Mpandaguta, Zivai Mupambireyi, Phyllis Mushati, Tinofa Mutevedzi, Reggie Mutsindiri, Joshua Ndlovu (deceased), Helen Owen, Monique Pereboom, Laura Robertson, Christina Schumacher, Nadine Schur, Tom Zhuwau, and the management and staff at BRTI and the BRTI laboratory. Most of all, the authors thank the people of Mutasa, Makoni and Nyanga districts for their participation in the Manicaland cohort since 1998.

Contributors All authors read and contributed to this manuscript. SG, GPG, OM and CAN designed the study and raised the funding. CAN, RM and SG collected data for the study and enrolled participants. CC and MS designed and conducted the qualitative studies. PRM, JMut and JMay conducted the laboratory procedures. AT, RR and JE undertook data management. CC, JE, SG, TH, PM, SM, RR, LS, MS and RS analysed the data. SG wrote the first draft of the report, to which all authors contributed.

Funding Core funding for the Manicaland Cohort has been provided by the Wellcome Trust (084401/Z/07/B and 069516/Z/02/Z). UNAIDS, WH0, the European Union, the World Bank, and the Bill and Melinda Gates Foundation have provided funds for aspects of the work.

Competing interests SG has shares in AstraZeneca and GSK.

Ethics approval Medical Research Council of Zimbabwe; Imperial College Research Ethics Committee.

Provenance and peer review Not commissioned; externally peer reviewed.

Data sharing statement Data from the Manicaland Cohort can be obtained from the project website (http://www.manicalandhivproject.org/data-access.html). Here we provide a core data set which contains a sample of sociodemographic, sexual behaviour and HIV testing variables from all six rounds of the main survey, as well as data used in the production of recent academic publications. If further data are required, a data request form must be completed (available to download from our website) and submitted to s.gregson@imperial.ac.uk. If the proposal is approved, we will send a data sharing agreement which must be agreed upon before we release the requested data.

Open Access This is an Open Access article distributed in accordance with the terms of the Creative Commons Attribution (CC BY 4.0) license, which permits others to distribute, remix, adapt and build upon this work, for commercial use, provided the original work is properly cited. See: http://creativecommons.org/ licenses/by/4.0/

(C) Article author(s) (or their employer(s) unless otherwise stated in the text of the article) 2017. All rights reserved. No commercial use is permitted unless otherwise expressly granted.

\section{REFERENCES}

1. Gregson S, Anderson RM, Ndlovu J, et al. Recent upturn in mortality in rural Zimbabwe: evidence for an early demographic impact of HIV1 infection? AIDS 1997;11:1269-80.

2. Gregson S, Mason PR, Garnett GP, et al. A rural HIV epidemic in Zimbabwe? Findings from a population-based survey. Int J STD AIDS 2001;12:189-96.

3. Gregson S, Adamson S, Papaya S, et al. Impact and process evaluation of integrated community and clinic-based HIV-1 control: a cluster-randomised trial in eastern Zimbabwe. PLoS Med 2007; 4 :e302.

4. Lopman B, Lewis J, Nyamukapa C, et al. HIV incidence and poverty in Manicaland, Zimbabwe: is HIV becoming a disease of the poor? AIDS 2007;21(Suppl 7):S57-S66.

5. Patel V, Simunyu E, Gwanzura F, et al. The Shona Symptom Questionnaire: the development of an indigenous measure of common mental disorders in Harare. Acta Psychiatr Scand 1997;95:469-75. 
6. Division of Mental Health, World Health Organization. A user's Guide to the Self-Reporting Questionnaire. Geneva, 1994:78.

7. Zaba B, Reniers G, Slaymaker E, et al. Understanding why people participate in HIV surveillance. Bull World Health Organ 2015;93:356-7.

8. Lopman BA, Barnabas RV, Boerma JT, et al. Creating and validating an algorithm to measure AIDS mortality in the adult population using verbal autopsy. PLoS Med 2006;3:e312-81.

9. Gregson S, Mushati P, Nyamukapa C. Adult mortality and erosion of household viability in AIDS-afflicted towns, estates, and villages in eastern Zimbabwe. J Acquir Immune Defic Syndr 2007;44:188-95.

10. Marsh KA, Nyamukapa CA, Donnelly CA, et al. Monitoring trends in HIV prevalence among young people, aged 15 to 24 years, in Manicaland, Zimbabwe. J Int AIDS Soc 2011;14:27.

11. Wringe $\mathrm{A}$, Church $\mathrm{K}$, Machiyama $\mathrm{K}$, et al. Identifying gaps in HIV service delivery across the diagnosis-to-treatment cascade: findings from health facility surveys in six sub-Saharan african countries. Vancouver: International AIDS Conference, 2015.

12. Aanensen DM, Huntley DM, Feil EJ, et al. EpiCollect: linking Smartphones to web applications for Epidemiology, Ecology and Community Data Collection. PLoS One 2009;4:e6968.

13. Gregson S, Garnett GP, Nyamukapa CA, et al. HIV decline associated with behaviour change in Eastern Zimbabwe. Science;2006:664-6.

14. Manzou R, Schumacher C, Gregson S. Temporal dynamics of religion as a determinant of HIV infection in East Zimbabwe: a serial crosssectional analysis. PLoS One 2014;9:e86060.

15. Eaton JW, Takavarasha FR, Schumacher CM, et al. Trends in concurrency, polygyny, and multiple sex partnerships during a decade of declining HIV prevalence in eastern Zimbabwe. J Infect Dis 2014;210(suppl_2):S562-S568.

16. Halperin DT, Mugurungi $O$, Hallett TB, et al. A surprising prevention success: why did the HIV epidemic decline in Zimbabwe? PLoS Med 2011;8:e1000414.

17. Pufall E, Eaton JW, Nyamukapa CA, et al. The relationship between parental education and children's schooling in a time of economic turmoil: The case of east Zimbabwe, 2001 to 2011. International Journal of Educational Development. 2016;5:125-134.

18. Chin J. Public health surveillance of AIDS and HIV infections. Bulletin of the World Health Organization 1990;68:529-36.

19. Zimbabwe Ministry of Health. HIV and AIDS surveillance: annual Report 1989: AIDS Control Programme, Zimbabwe Ministry of Health, 1989

20. Gregson S, Zhuwau T, Anderson RM, et al. Age and religion selection biases in HIV-1 prevalence data from antenatal clinics in Manicaland, Zimbabwe. Cent Afr J Med 1995;41:339-45.

21. Gregson S, Terceira N, Kakowa M, et al. Study of bias in antenatal clinic HIV-1 surveillance data in a high contraceptive prevalence population in sub-Saharan Africa. AIDS 2002;16:643-52.

22. Gregson S, Dharmayata K, Pereboom M, et al. Do HIV prevalence trends in ANC surveillance represent trends in the general population in sub-Saharan Africa? AIDS 2015;29:1845-53.

23. Walker N, Stanecki KA, Brown T, et al. Methods and procedures for estimating HIV/AIDS and its impact: the UNAIDS/WHO estimates for the end of 2001. AIDS 2003;17:2215-25.

24. Stover J, Andreev K, Slaymaker E, et al. Updates to the Spectrum model to estimate key HIV indicators for adults and children. AIDS 2014;28(Suppl 4):S427-S434.

25. Zimbabwe Ministry of Health and Child Welfare. Zimbabwe National HIV and AIDS estimates 2013. Harare, 2014.

26. Silhol R, Gregson S, Nyamukapa C, et al. Empirical validation of the UNAIDS Spectrum model for subnational HIV estimates: case-study of children and adults in Manicaland, Zimbabwe. AIDS 2017;31 Suppl 1:S41-S50.

27. Catania JA, Gibson DR, Chitwood DD, et al. Methodological problems in AIDS behavioral research: influences on measurement error and participation bias in studies of sexual behavior. Psychol Bull 1990;108:339-62.

28. Gregson S, Mushati P, White PJ, et al. Informal confidential voting interview methods and temporal changes in reported sexual risk behaviour for HIV transmission in sub-Saharan Africa. Sex Transm Infect 2004;80(suppl_2):ii36-ii42.

29. Gregson S, Nyamukapa CA, Garnett GP, et al. Sexual mixing patterns and sex-differentials in teenage exposure to HIV infection in rural Zimbabwe. Lancet 2002;359:1896-903

30. Smith J, Nyamukapa C, Gregson S, et al. The distribution of sex acts and condom use within partnerships in a rural sub-Saharan African population. PLoS One 2014;9:e88378.

31. Hallett TB, Gregson S, Lewis JJ, et al. Behaviour change in generalised HIV epidemics: impact of reducing cross-generational sex and delaying age at sexual debut. Sex Transm Infect 2007:83:i50-i54.
32. Lopman B, Garnett GP, Mason PR, et al. Evidence of absence: injection history and HIV incidence in Rural Zimbabwe. Public Library of Science Medicine 2005;2:142-6.

33. Sherr L, Lopman B, Kakowa M, et al. Voluntary counselling and testing: uptake, impact on sexual behaviour, and HIV incidence in a rural Zimbabwean cohort. AIDS 2007;21:851-60.

34. Gregson S, Nyamukapa CA, Schumacher C, et al. Did national HIV prevention programmes contribute to HIV decline in eastern Zimbabwe? evidence from a prospective community survey. Sex Transm Infect 2011;38(6):475-482.

35. Cremin I, Nyamukapa C, Sherr L, et al. Patterns of Self-reported Behaviour Change Associated with Receiving Voluntary Counselling and Testing in a Longitudinal Study from Manicaland, Zimbabwe. AIDS Behav 2010;14:708-15.

36. Coffee MP, Garnett GP, Mlilo M, et al. Patterns of movement and risk of HIV infection in rural Zimbabwe. J Infect Dis 2005;191(s1):S15 9-S167.

37. Mundandi C, Vissers $\mathrm{D}$, Voeten $\mathrm{H}$, et al. No difference in HIV incidence and sexual behaviour between out-migrants and residents in rural Manicaland, Zimbabwe. Trop Med Int Health 2006;11:705-11.

38. UNAIDS. Evidence for HIV decline in Zimbabwe: a Comprehensive Review of the Epidemiological Data. Geneva: UNAIDS, 2005.

39. Anderson RM, May RM, McLean AR. Possible demographic consequences of AIDS in developing countries. Nature $1988 ; 332: 228-34$

40. United Nations. The AIDS Epidemic and its Demographic Consequences: United Nations. New York, 1991

41. Gregson S, Garnett GP, Anderson RM. Assessing the Potential Impact of the HIV-1 Epidemic on Orphanhood and the Demographic Structure of Populations in sub-Saharan Africa. Popul Stud 1994:48:435-58.

42. Gregson S, Nyamukapa C, Lopman B, et al. Critique of early models of the demographic impact of HIV/AIDS in sub-Saharan Africa based on contemporary empirical data from Zimbabwe. Proc Natl Acad Sci U S A 2007;104:14586-91.

43. Zaba B, Terceira N, Mason P, et al. The contribution of HIV to fertility decline in rural Zimbabwe, 1985-2000. Popul Stud 2003;57:149-64.

44. Watts $\mathrm{H}$, Lopman B, Nyamukapa $\mathrm{C}$, et al. Rising incidence and prevalence of orphanhood in Manicaland, Zimbabwe, 1998 to 2003. AIDS 2005;19:717-25.

45. Gregson S, Nyamukapa CA, Garnett GP, et al. HIV infection and reproductive health in teenage women orphaned and made vulnerable by AIDS in Zimbabwe. AIDS Care 2005;17:785-94.

46. Watts H, Gregson S, Saito S, et al. Poorer health and nutritional outcomes in orphans and vulnerable young children not explained by greater exposure to extreme poverty in Zimbabwe. Trop Med Int Health 2007:12:584-93.

47. Nyamukapa CA, Gregson S, Lopman B, et al. HIV-associated orphanhood and children's psychosocial distress: theoretical framework tested with data from Zimbabwe. Am J Public Health 2008;98:133-41.

48. Reniers G, Slaymaker E, Nakiyingi-Miiro J, et al. Mortality trends in the era of antiretroviral therapy: evidence from the ALPHA Network. AIDS 2014;28:s533-s42.

49. Schur N, Mylne A, Mushati P, et al. The effects of household wealth on HIV prevalence in Manicaland, Zimbabwe. J Int AIDS Soc 2015;18:20063

50. Nhamo-Murire M, Campbell C, Gregson S. Community group membership and stigmatising attitudes towards people living with HIV in Eastern Zimbabwe. J Community Health 2014;39:72-82.

51. Skovdal M, Campbell C, Madanhire C, et al. Masculinity as a barrier to men's use of HIV services in Zimbabwe. Global Health 2011;7:13.

52. Skovdal M, Campbell C, Nyamukapa C, et al. When masculinity interferes with women's treatment of HIV infection: a qualitative study about adherence to antiretroviral therapy in Zimbabwe. J Int AIDS Soc 2011;14:29.

53. Gregson S, Zhuwau T, Anderson RM, et al. Apostles and Zionists: the influence of religion on demographic change in rural Zimbabwe. Popul Stud 1999;53:179-93.

54. Elmes J, Nhongo $\mathrm{K}$, Ward $\mathrm{H}$, et al. The price of sex: condom use and the determinants of the price of sex among female sex workers in eastern Zimbabwe. J Infect Dis 2014;210(Suppl 2):S569-S578.

55. Gregson S, Mushati P, Grusin H, et al. Social capital and women's reduced vulnerability to HIV infection in rural Zimbabwe. Popul Dev Rev 2011;37:333-59.

56. Gregson S, Nyamukapa CA, Sherr L, et al. Grassroots community organizations' contribution to the scale-up of HIV testing and counselling services in Zimbabwe. AIDS 2013;27:1657-66.

57. Campbell C, Scott K, Nhamo M, et al. Social capital and HIV competent communities: the role of community groups in managing 
HIV/AIDS in rural Zimbabwe. AIDS Care 2013;25(Suppl1):S114 $-\mathrm{S} 122$.

58. Campbell C, Scott K, Mupambireyi Z, et al. Community resistance to a peer education programme in Zimbabwe. BMC Health Serv Res 2014;14:574.

59. Skovdal M, Mushati P, Robertson L, et al. Social acceptability and perceived impact of a community-led cash transfer programme in Zimbabwe. BMC Public Health 2013;13:342.

60. Maher D, Biraro S, Hosegood V, et al. Collaborators in ALPHA Network. Translating global health research aims into action: the example of the ALPHA network. Trop Med Int Health 2010;15:321-8.

61. Gregson S, Zhuwau T, Ndlovu J, et al. Methods to reduce social desirability bias in sex surveys in low-development settings: experience in Zimbabwe. Sex Transm Dis 2002;29:568-75.

62. Hallett TB, Gregson S, Kurwa F, et al. Measuring and correcting biased child mortality statistics in countries with generalized epidemics of HIV infection. Bull World Health Organ 2010;88:761-8.

63. Gregson S, Gonese E, Hallett TB, et al. HIV decline in Zimbabwe due to reductions in risky sex? Evidence from a comprehensive epidemiological review. Int J Epidemiol 2010;39:1311-23.

64. Hallett TB, Gregson S, Mugurungi O, et al. Is there evidence for behaviour change affecting the course of the HIV epidemic in Zimbabwe? A new mathematical modelling approach. Epidemics 2009;1:108-17.
65. Gregson S, Todd J, Zaba B. Sexual behaviour change in countries with generalised HIV epidemics? Evidence from population-based cohort studies in sub-Saharan Africa. Sex Transm Infect 2009;85 Suppl 1:i1-i2.

66. Reniers G, Wamukoya M, Urassa M, et al. Data Resource Profile: Network for Analysing Longitudinal Population-based HIV/AIDS data on Africa (ALPHA Network). Int J Epidemiol 2016;45:83-93.

67. Hallett TB, et al. Declines in HIV prevalence can be associated with changing sexual behaviour in Uganda, urban Kenya, Zimbabwe, and urban Haiti. Sex Transm Infect 2006;82:11-i8.

68. Robertson L, Mushati P, Eaton JW, et al. Effects of unconditional and conditional cash transfers on child health and development in Zimbabwe: a cluster-randomised trial. Lancet 2013;381:1283-92.

69. Voeten H, Vissers DCJ, Gregson S, et al. Ecological association between migration and HIV prevalence in african cities. Sexually Transmitted Diseases 2010;37:240-3.

70. Pufall EL, Gregson S, Eaton JW, et al. The contribution of schools to supporting the well being of children affected by HIV in Eastern Zimbabwe. AIDS 2014;28(Suppl 3):S379-S387.

71. Campbell C, Andersen L, Mutsikiwa A, et al. Factors shaping the HIV-competence of two primary schools in rural Zimbabwe. Int $J$ Educ Dev 2015;41:226-36.

72. Melegaro A, Del Fava E, Poletti P, et al. Investigating social contact structures and time use in sub-Saharan Africa. Public Library of Science One 2017;12(1): e0170459. 